\title{
Publicidade de Alimentos e Escolhas Alimentares de Crianças ${ }^{1}$
}

\author{
Marcia Hiroko Ueda ${ }^{2}$ \\ Rafael Barreiros Porto \\ Láercia Abreu Vasconcelos \\ Universidade de Brasília
}

\begin{abstract}
RESUMO - O efeito de vídeos de desenhos com publicidade de alimentos saudáveis e não saudáveis versus vídeos neutros sobre as escolhas alimentares foi avaliado em 24 crianças de escola pública. Os vídeos foram apresentados em cinco sessões, sendo cada grupo exposto a uma sequência específica de publicidade. Após a exposição, a criança escolhia figuras de alimentos para as refeições do dia. Análises intra e entre sujeitos, por meio da Ancova de medidas repetidas, demonstraram que, com a mudança de vídeo, as crianças alteraram em até $13 \%$ a escolha de alimentos saudáveis ou não. O aumento da exibição de publicidade de alimentos saudáveis e a diminuição da publicidade de alimentos não saudáveis pode contribuir para a alimentação e peso saudáveis.
\end{abstract}

Palavras-chave: publicidade de alimentos, práticas alimentares, estado nutricional, análise funcional, contingência, crianças

\section{Food Advertising and Children's Food Choices}

\begin{abstract}
The effect of cartoon videos with advertisements of healthy and unhealthy food versus neutral videos on food choices was assessed in 24 children from a public school. The videos were presented in 5 sessions. Each group was exposed to a specific sequence of advertisement. After the exposure of the videos, the child chose food figures for daily meals. Within and between subject analyses, performed by repeated measures ANCOVA, showed that with change of videos, the children altered by up to $13 \%$ their choices of healthy or unhealthy food. The increase of healthy food advertising and the decrease of unhealthy food advertising may contribute for healthy food and weight.
\end{abstract}

Keywords: food advertisement, eating habits, nutritional status, functional analysis, contingency, children

A mídia televisiva tem sido utilizada como um amplo meio de comunicação voltado para a promoção de alimentos não saudáveis, havendo pouco espaço para a promoção de práticas saudáveis de alimentação (Dixon, Scully, Wakefield, White, \& Crawford, 2007). A mídia televisiva tem ocupado, no dia a dia das crianças, mais de $50 \%$ do seu tempo em comparação com outras atividades tais como tarefas escolares e familiares, além de brincadeiras (Groebel, 2002). Segundo a Pesquisa Nacional por Amostra de Domicílios (PNAD), em 2007, 94,5\% dos domicílios tinham televisão em suas casas (IBGE, 2008). A maioria das crianças e dos adolescentes preferia a televisão, os jogos eletrônicos e o cinema como forma de diversão, e o jovem brasileiro permanecia, em média, de três a quatro horas diante da TV (Ministério da Justiça, 2009).

Ao se divertirem em frente à televisão, crianças e jovens são expostos à publicidade de marcas. $\mathrm{O}$ trabalho publicitário divulga uma marca, usando recursos visuais e auditivos, e geralmente exagera nos elogios à marca com o objetivo de criar demanda pelo produto e gerar lucro para o anunciante (Cohen, 2005). Promove o consumo, utilizando informações na tentativa de mobilizar a fantasia. Logo, aquilo que é divulgado referente às marcas pode gerar ilusão, incentivar

1 Apoio: CAPES

2 Endereço para correspondência: Centro de saúde 01 de Planaltina. Área especial vias NS/WL4, Planaltina, DF. CEP 73310-000.

E-mail: marcia.ueda@gmail.com comportamentos superticiosos ou inapropriados (Coelho, 1994). Alerta-se que a publicidade não é nociva por si só, sendo um meio de divulgação legítimo em países que têm economia aberta e livre (Nunes, 2011).

Porto (2011) revelou que vídeos publicitários podem conter situações que demonstram ocorrência de reforço e punição na aquisição ou não de um produto, favorecendo a compra do produto anunciado. Logo, a análise minuciosa de vídeos publicitários veiculados na mídia é fundamental para a compreensão dos comportamentos induzidos pelos anunciantes.

As pesquisas voltadas para a avaliação das escolhas de compra do consumidor podem auxiliar a identificar o impacto da publicidade no consumo e abrir caminho para ajustes em suas veiculações no dia a dia dos brasileiros. Foxall (2004) sugere que eventos ambientais antecedentes ao comportamento de compra, como a exposição à publicidade, podem influenciar as escolhas de consumo, mas que isso pode depender da história de reforçamento que cada consumidor presenciou. Porto e Oliveira-Castro (2013), por sua vez, encontraram empiricamente que a publicidade poderia ter efeitos isolados sobre a correspondência entre a última compra, a declaração de compra e a compra subsequente de marcas, mas que geralmente a publicidade interage com a história de reforçamento dos consumidores quando eles são expostos a diferentes níveis de magnitude de reforço utilitário.

Diante dos efeitos da publicidade de marcas nas compras, a compreensão de suas características específicas (e.g., tipo 
de produto veiculado, a mídia utilizada para as veiculações, uso de imagens persuasivas, etc) pode explicar melhor seus efeitos nas escolhas dos consumidores, como aquelas relacionadas a alimentos saudáveis ou não saudáveis. $\mathrm{O}$ monitoramento das publicidades no Distrito Federal, realizado em dois canais abertos e dois canais de assinatura, revelou que $96,7 \%$ das publicidades se referiam a alimentos classificados como não saudáveis, ou seja, com excesso de gordura, açúcares e sal, como fast food, doces, sorvetes, refrigerantes e sucos industrializados, e apenas 3,3\% foram de alimentos saudáveis como cereais, carnes, pães, massas (Monteiro, 2009). Os alimentos não saudáveis anunciados nas publicidades são caracterizados como práticos, pois $82,2 \%$ são para consumo imediato, facilitando a preparação das refeições (Nascimento, 2006). Além disso, de acordo com a Agência Nacional de Vigilância Sanitária - ANVISA (2007), o consumo desses alimentos tem sido estimulado pela associação de personagens de desenhos infantis, artistas famosos, brindes e coleções de brinquedos. Esses fatores podem contribuir para o aumento das taxas de obesidade e de outras doenças, em idade posterior ou até mesmo durante a infância, pois grande parte desses alimentos é classificada com alta densidade energética e baixo valor nutritivo (Almeida, Nascimento, \& Quaioti, 2002).

A genética, os aspectos socioambientais (e.g., práticas alimentares) e a atividade física têm sido relacionados ao estado nutricional (e.g., Mendonça \& Anjos, 2004). Além disso, a televisão tem sido apontada como um fator importante para a obesidade. Diante disso, alguns estudos relacionam exposição a conteúdos televisivos e nível de atividade física com a obesidade (e.g., Andersen, Crespo, Bartlett, Cheskin, \& Pratt, 1998; Jago, Baranowski, Baranowski, \& Greaves, 2005). Entretanto, Veerman, Van Beeck, Barendregt e Mackenbach (2009) e Zimmerman e Bell (2010) têm mostrado que a presença de publicidade de alimentos não saudáveis na televisão é, por si mesma, relacionada à obesidade, a despeito do sedentarismo.

Ressalta-se que a mídia pode ser um meio para a promoção da alimentação saudável ao veicular alimentos saudáveis e mensagens nutricionais acuradas (Byrd-Bredbenner \& Grasso, 2000). Peterson, Jeffrey, Bridgwater e Dawson (1984) utilizaram vídeos com programas sobre alimentação saudável, de 20 minutos por 10 dias, para avaliar as práticas alimentares de crianças de 5 e 6 anos. A aprendizagem sobre nutrição foi observada e também uma pequena tendência para o consumo de alimentos saudáveis.

Entretanto, a maioria dos estudos relacionando a mídia e o comportamento alimentar de crianças encontram dados desfavoráveis à saúde. Os estudos abordam as preferências alimentares não saudáveis, o sobrepeso, a obesidade e o estímulo ao consumo. Vilaça, Paixão e Pordeus (1997) mostram que as crianças com idade inferior a 5 anos são influenciadas pelos comerciais e seus efeitos especiais, adotando práticas e estereótipos veiculados pela mídia. Além disso, as crianças com maior tempo de exposição à mídia televisiva adquirem preferência por produtos mais nocivos à saúde em comparação com as crianças menos expostas a esse meio de comunicação.

Com o objetivo de avaliar a relação entre tempo de exposição à televisão e preferência por marcas mais conhecidas, Robinson, Borzekowski, Matheson e Kraemer (2007) testa- ram cinco pares idênticos de alimentos e bebidas, embalados com marca de um fast food conhecido ou embalados sem marca. Foi observada preferência pela marca conhecida, com maior efeito da marca sobre o comportamento das crianças que assistiam mais televisão e que consumiam o produto com maior frequência. Em um estudo comparável, Borzekowski e Robinson (2001) avaliaram o efeito da publicidade de alimentos com crianças de 2 a 6 anos que assistiam a um vídeo de desenho com ou sem os comerciais. As crianças que assistiram ao vídeo com os comerciais escolhiam mais a figura dos produtos apresentados na publicidade do que as crianças do grupo controle.

Dixon et al. (2007) avaliaram também a relação entre publicidade e preferências alimentares de crianças, mas com idade de 10 a 11 anos. Quatro grupos assistiram a vídeos diferentes: somente publicidade saudável; somente publicidade não saudável; com ambas publicidades; ou com nenhuma publicidade. Assistir televisão e publicidades não saudáveis apresentaram correlação positiva com o relato de consumo de alimentos não saudáveis. Da mesma maneira, as publicidades de alimentos saudáveis originaram relatos de consumo desses alimentos. Esses resultados são consistentes com os de Boynton-Jarret et al. (2003), os quais acompanharam adolescentes em um período de 19 meses. Foi observada uma relação inversa entre o tempo de exposição à televisão e o consumo de frutas e verduras, sugerindo a substituição dos alimentos saudáveis pelos não saudáveis amplamente presentes na televisão.

Halford et al. (2007) investigaram a escolha e o consumo de alimentos relacionados à exposição à publicidade e ao estado nutricional de crianças. Crianças expostas a desenhos com publicidade de alimentos apresentaram maior ingestão calórica comparadas àquelas expostas a desenhos com publicidade de brinquedo. As crianças com obesidade e sobrepeso foram as mais responsivas à publicidade de alimentos, escolhendo e consumindo lanches com maior densidade energética. Em estudo semelhante, o reconhecimento das marcas das publicidades de alimentos do vídeo assistido foi maior para as crianças com sobrepeso e obesidade em comparação com as crianças eutróficas ${ }^{3}$ (Halford, Gillespie, Brown, Pontin, \& Dovey, 2004).

A essa literatura se somam estudos desenvolvidos no Brasil, os quais investigaram as relações entre exposições à mídia ${ }^{3}$ com alimentos não saudáveis e o seu consumo. Halpern (2003) avaliou os comerciais veiculados em programação infanto-juvenil de canais abertos da televisão, sendo observada maior presença de publicidade de alimentos não saudáveis. Foi observado o consumo desses alimentos no lanche e pedidos desses alimentos aos pais. O trabalho de Nascimento (2006) também identificou grande presença dos alimentos não saudáveis na televisão, interferindo na decisão de compra e consumo dos alimentos e favorecendo o aumento da obesidade. Monteiro (2009), por sua vez, realizou um experimento para avaliar a influência da publicidade de alimentos sobre o consumo de alimentos saudáveis e não saudáveis, utilizando metodologia semelhante à do estudo de Dixon et al. (2007), com publicidades de marcas conhecidas,

3 O termo eutrófico é utilizado para classificar indivíduos de peso adequado (Halford, Gillespie, Brown, Pontin, \& Dovey, 2004). 
e avaliando o comportamento a partir de questionários. Nesse estudo não foram encontradas diferenças significativas nas variáveis relacionadas ao consumo alimentar nos grupos que foram expostos à publicidade de alimentos saudáveis e não saudáveis, ambos os tipos de publicidade ou publicidade de brinquedos.

Além da participação da mídia no processo de construção das práticas alimentares, ressalta-se a participação dos pais enquanto modelos a serem seguidos. Hursti (1999) observou que um alimento desconhecido para a criança tende a ser mais aceito quando ela observa a mãe consumindo-o do que uma pessoa estranha. A publicidade reconhece o papel não somente dos pais, mas também de amigos, personagens de desenhos, artistas e mascotes nas escolhas realizadas por crianças (Halpern, 2003). Assim, uma estratégia utilizada em peças publicitárias é mostrar o personagem comendo o alimento anunciado, demonstrando prazer, alegria e diversão (Nascimento, 2006). Em alguns programas infantis, os produtos não saudáveis são mais enfatizados, sendo valorizados e consumidos em excesso pelos personagens (Radnitz et al., 2009).

Os estudos mencionados foram realizados com grupos, não sendo investigado simultaneamente as influências da publicidade intra e entre sujeitos. Além disso, não investigaram o efeito da exposição a um vídeo neutro, vídeo com alimentos saudáveis e vídeo com alimentos não saudáveis para o mesmo sujeito. A maioria dos estudos apresentava marcas conhecidas, o que pode enviesar as escolhas, pois a criança poderia optar por produtos já conhecidos e consumidos por ela em seu ambiente natural. No Brasil, além dos pontos citados, não foi identificado nenhum estudo em que a avaliação das escolhas alimentares simulava situações reais de escolha, pois avaliaram mudanças no consumo a partir da aplicação de questionário, fora de um contexto de consumo de alimentos .

Assim, este estudo propôs um delineamento experimental diferenciado: a replicação intra e entre sujeito. Neste estudo, procurou-se observar o efeito de vídeos (vídeos neutros, vídeos com alimentos saudáveis e vídeos com alimentos não saudáveis) sobre o comportamento de escolha de cada criança, avaliado em cinco sessões sequenciais, alterando os tipos de vídeo. As marcas veiculadas nas publicidades eram desconhecidas, garantindo o controle do efeito das marcas. Outro diferencial proposto foi a avaliação do comportamento alimentar por meio de um jogo composto por ímãs com figuras de alimentos que estavam disponíveis para a criança selecionar, simulando as escolhas dos alimentos para as refeições do dia. Dessa maneira, a simulação se aproximou das situações vivenciadas no dia a dia pelas crianças.

\section{Método}

\section{Participantes}

A pesquisa foi realizada de acordo com a aprovação do Comitê de Ética da Faculdade de Ciências da Saúde da Universidade de Brasília (Processo 031/09).
A amostra consistiu de 24 crianças de escola pública do Distrito Federal, com idade entre 7 a 9 anos. A distribuição segundo o gênero e idade foi a seguinte: duas meninas e cinco meninos com 7 anos; sete meninas e um menino com 8 anos e três meninas e seis meninos com 9 anos. As crianças foram convidadas a participar da pesquisa por meio do Termo de Consentimento que foi enviado aos responsáveis. O critério de inclusão foi baseado na distribuição por gênero e na inexistência de diagnóstico de doença

Dois grupos com 12 crianças, composto por número igual de meninos e meninas foram formados de acordo com a ordem de exposição a três tipos de vídeos - neutros (VN), com alimentos saudáveis (VS) e com alimentos não saudáveis (VNS). Para o cálculo do poder amostral multiplicou-se o número de participantes (24 crianças) pelo número de ocasiões de medidas repetidas (cinco sessões). Logo, 120 observações foram obtidas, sendo 60 em um grupo (VN-VS-VS-VNS-VNS) e 60 em outro (VN-VNS-VNS-VS-VS). Foi utilizado o teste da família $\mathrm{F}$ - Anova de medidas repetidas com interações intra e entre sujeitos, com um tamanho do efeito médio $\mathrm{f}=0,25$ e com $\alpha=0,05$. O resultado demonstrou um $\mathrm{F}$ crítico $=2,5 \mathrm{com}$ um poder $=87 \%$, sendo suficiente para minimizar o Erro Tipo 2.

\section{Instrumento}

Para caracterização da amostra foi aplicado um questionário e avaliado o estado nutricional da criança. O questionário continha perguntas sobre os dados sociodemográficos, questões sobre a televisão (número de horas, acompanhamento dos pais), atividade física e alimentação da criança. O estado nutricional da criança foi avaliado pelo cálculo do Índice de Massa Corporal (IMC), que divide o peso, em quilos, pelo quadrado da altura, em metros. A partir desse índice, a criança foi classificada como: baixo peso, eutrófica, excesso de peso ou obesidade, de acordo com os pontos de corte do IMC para idade e sexo (Conde \& Monteiro, 2006). O peso foi aferido em balança com capacidade de $150 \mathrm{~kg}$ e precisão de $100 \mathrm{~g}$ e a altura no estadiômetro.

Os vídeos utilizados neste estudo foram editados a partir do programa Adobe Premiere ProCS3 e exibidos em um computador portátil. Os vídeos foram compostos por desenho infantil educativo, que não menciona e nem exibe alimentos, com duração de cerca de cinco minutos, e por cinco minutos de publicidade de alimentos inseridas no desenho em cinco intervalos. Os vídeos neutros foram compostos por informações educativas, sem objetivo comercial - a preservação da natureza, da água, cuidados com o trânsito, cuidado com acidentes domésticos, higiene pessoal, cuidados com o livro, com a escola e o incentivo à prática de esportes. Os vídeos com publicidade foram elaborados com base em vídeos exibidos no passado no país e em outros países, sendo as marcas de produtos conhecidos substituídas por uma marca desconhecida. Nos vídeos com publicidade de alimentos foram exibidos dois alimentos por intervalo. Dentre as publicidades de alimentos saudáveis foram exibidos, no primeiro intervalo, leite e cereal integral sem açúcar; no segundo, frutas com suco de fruta e biscoito cream cracker integral; no terceiro um prato saudável com arroz, feijão, salada, carne e hortaliças e 
verduras; no quarto, macarrão e frango; e, no quinto, biscoito Maria e pão integral. Dentre as publicidades de alimentos não saudáveis foram exibidos, no primeiro intervalo, cereal açucarado e refresco em pó; no segundo, biscoito recheado e salgadinho; no terceiro, hambúrguer com cachorro quente e refrigerante; no quarto, empanado de frango e macarrão instantâneo; e, no quinto, sobremesas com chocolate e pizza. A ordem de apresentação das publicidades foi a mesma para a segunda exibição do vídeo saudável ou não saudável.

O "jogo de escolhas", denominação da tarefa a ser desenvolvida pelas crianças, foi composto por um tabuleiro de metal $(80 \mathrm{~cm}$ x $50 \mathrm{~cm})$, onde foram colocados os cenários: imagem de uma prateleira de mercado e mesa com cadeira para a refeição. Sobre a imagem da prateleira eram colocados os ímãs com figuras de 36 alimentos $(4 \mathrm{~cm} \mathrm{x} 4 \mathrm{~cm})$, sendo a metade saudável - os apresentados na publicidade saudável acrescido de: leite, iogurte, queijo, pão branco, batata cozida, salgado assado - e a outra metade não saudável - os apresentados na publicidade não saudável acrescido de: suco de caixa, salgado frito, batata-frita, linguiça, guloseimas (bala, chiclete, pirulitos), margarina. As escolhas foram filmadas em câmera e preenchidas na folha de registro (alimentos escolhidos nos contextos café da manhã, almoço, lanche e jantar).

\section{Procedimento}

O questionário e o Termo de Consentimento foram enviados aos responsáveis por meio da criança. Questionários incompletos foram preenchidos por telefone. A pesquisa foi realizada em sala disponibilizada pela direção da escola. Um dos pesquisadores foi responsável pela avaliação nutricional, apresentação dos vídeos e coordenação do jogo em todas as sessões. No primeiro dia de sessão, o peso da criança foi aferido com uniforme escolar, sem casaco e descalça. A medição da altura foi realizada também com a criança descalça e com a nuca, as nádegas e os calcanhares encostados à parede. Depois a criança foi convidada a sentar-se à mesa e assistir a um desenho.

A coleta de dados ocorreu em diferentes momentos do turno escolar, em momento mais conveniente para que a criança, evitando a perda de conteúdo em sala de aula. A criança poderia ter lanchado ou não. Observações das sessões sugerem que não houve menor ou maior seleções de alimentos controladas pelo lanche escolar.

A $1^{a}$ sessão foi a de linha de base e tinha como objetivo investigar as escolhas de alimentos após um vídeo neutro. Nas $2^{\mathrm{a}}$ e $3^{\mathrm{a}}$ sessões foram apresentados os vídeos com publicidade de alimentos saudáveis (ou de alimentos não saudáveis) e nas $4^{\mathrm{a}}$ e $5^{\mathrm{a}}$ sessões, as crianças que iniciaram com o vídeo com publicidade saudável assistiram ao vídeo com publicidade não saudável e vice-versa. Após o vídeo, a pesquisadora posicionava o jogo de escolhas em frente da criança e apresentava a instrução:

Você está vendo estes alimentos? Imagine que são de verdade. Agora você vai escolher alimentos que você comeria e beberia em seu café da manhã, almoço, lanche e jantar. Aqui está a mesa e a cadeira onde vai colocar os alimentos que vai comer e beber. Não existe escolha certa ou errada. Não se preocupe se escolher muito ou pouco, se é o mesmo alimento e bebida da outra vez ou se é diferente. O importante é escolher realmente o que você quer comer e beber. Entendeu? (ver se a criança compreendeu). Temos os seguintes alimentos (citar todos). Se fosse a hora do seu café da manhã. Coloque sobre a mesa o que você quer comer e beber para o café da manhã. (Esperar escolha). Agora está na hora de seu almoço. Coloque sobre a mesa o que você quer comer e beber no seu almoço. (Esperar escolha). Agora, na hora de um lanche. Você tem esses mesmos alimentos. Coloque sobre a mesa o que você quer comer e beber no seu lanche. (Esperar escolha). Agora está na hora do seu jantar. Coloque sobre a mesa o que você quer comer e beber no seu jantar. (Esperar escolha).

Caso a criança realizasse alguma pergunta, esta era respondida desde que fosse relacionada à instrução do jogo, não sendo repassadas informações extras sobre o procedimento.

Ao final de cada sessão, a pesquisadora agradecia a participação da criança com adesivos. No último dia, como agradecimento, as crianças escolheram lembranças como: lapiseiras, caderninhos, garrafas de água, carrinhos, bolsinhas.

\section{Análise de dados}

Os dados foram analisados por medidas de tendência central - médias e desvios padrões - seguidos por medidas estatísticas inferenciais. Realizou-se uma análise de covariância intra e entre sujeitos (Ancova de medidas repetidas) para detectar se os grupos de crianças (entre sujeitos) expostos a cada uma das cinco sessões (intrassujeitos) com as manipulações dos vídeos de publicidade escolhiam percentualmente mais ímãs com alimentos saudáveis do que não saudáveis. Foram controladas estatisticamente algumas variáveis individuais que poderiam exercer influência nessa relação, a saber: valor do IMC, tempo médio que a criança assistia à televisão por semana, idade em meses das crianças e estado nutricional.

Para viabilizar o teste Ancova de medidas repetidas, foi feito o teste Kolmogorov-Smirnov para testar a normalidade da variável dependente, isto é, o percentual de escolha de alimentos saudável em relação ao não saudável para cada sessão. Em seguida, foi feito o teste $\mathrm{M}$ de Box para averiguar a homogeneidade da variância. Como havia um pequeno número de participantes em cada grupo - 12 crianças, foi utilizado Huynh-Feldt Epsilon $(0,854)$ como ajuste do teste Greenhouse-Geisser, que corrige o teste quando a esfericidade é violada.

\section{Resultados}

\section{Avaliação nutricional e questões sobre alimentação, atividade física e hábitos de televisão}

A avaliação do estado nutricional das crianças, segundo o IMC por idade mostrou que $58,4 \%$ das crianças estavam eutróficas, $33,3 \%$ com sobrepeso e $8,3 \%$ apresentaram obesidade. 
Em relação ao consumo de alimentos foi relatado no questionário o consumo de arroz, feijão, leite, pão, açúcar, frutas, achocolatado e margarina, com uma frequência de cinco vezes ou mais por semana. O consumo de frutas, verduras e legumes foi adequado, respectivamente, para 53\%, $46 \%$ e $43 \%$ das crianças.

Entre as intervenções dos pais, utilizadas no contexto da alimentação, estão o uso de atenção; a disponibilidade de outros alimentos saudáveis; descrições de contingências (i.e., consumo de alimentos saudáveis e seus potenciais efeitos); inclusão de alimentos não preferidos, de forma mascarada, na refeição da criança; uso de modelos (e.g., pais e amigos); alimentação atrativa: variação e cor dos alimentos.

Quanto à prática de atividade física, 58,3\% praticavam algum esporte, sendo em número igual de meninos e meninas.

Em relação à exposição à televisão foi observado que, durante a semana, o mínimo de horas diárias foi de $1 \mathrm{~h}$ e o máximo foi de $7 \mathrm{~h}(\dot{\mathrm{x}}=2,8, \mathrm{DP}=1,7)$, e aos finais de semana, o mínimo foi de $1 \mathrm{~h}$ e o máximo foi de $11 \mathrm{~h}(\dot{\mathrm{x}}=$ $4,2$, DP $=2,7)$. Foram encontradas diferenças entre meninos e meninas apenas aos finais de semana, sendo maior a exposição entre as meninas comparado aos meninos $(4,4$ $\mathrm{h}$ versus $4,0 \mathrm{~h}$ ). Os eutróficos assistiram, em média, 3,7 h; os com sobrepeso, em média, $4,2 \mathrm{~h}$ e os obesos, em media, 10,5 h. Em relação ao acompanhamento da criança durante a exposição à televisão, foi observado que cinco crianças estavam sozinhas enquanto assistiam à televisão $(20,8 \%)$, 10 crianças estavam sempre acompanhadas $(41,7 \%)$ e nove crianças $(37,5 \%)$ ora assistiam à televisão sozinhas ora acompanhadas por membros da família (pais, tia, irmão) ou empregada doméstica.

Um total de $71 \%$ das crianças assistia à televisão durante as refeições. Analisando de acordo com o estado nutricional, $100 \%$ das crianças classificadas como obesas, $75 \%$ das classificadas com sobrepeso e $64 \%$ das classificadas como eutróficas assistiam à televisão durante as refeições. Ao considerar a participação das crianças em compras, de acordo com as respostas dos pais, esta tem sido realizada por $62,5 \%$ das crianças, sendo os biscoitos, iogurte, sucos de caixa, refresco, bolo, salgadinho, sorvete, chocolate, mas também frutas, os alimentos escolhidos por elas.

\section{Efeitos da publicidade na escolha de alimentos}

Os testes realizados para avaliar a confiança nos parâmetros estatísticos resultantes da análise da ANCOVA de medidas repetidas foram satisfeitos. $\mathrm{O}$ resultado sugeriu que a variável em cada sessão teve uma distribuição aproximadamente normal, variando entre $Z=0,61 ; p>0,05$ até $Z=0,70 ; p>0,05$. $O$ teste $M$ de Box $(M=20,8 ; p>$ $0,05)$ indicou que não existe violações do pressuposto da homogeneidade das matrizes de variância-covariância entre os grupos. Porém, o pressuposto de esfericidade não foi satisfeito (Mauchly's $\mathrm{W}=0,17 ; \mathrm{p}<0,01$ ). Os resultados mostraram ser improvável que as diferenças entre as sessões tenham se originado somente em virtude do erro amostral $[\mathrm{F}(3,47)=3,4 ; \mathrm{p}=0,02] . \mathrm{O}$ tamanho de efeito $\left(\eta^{2}\right.$ parcial $=$ $0,196)$ mostrou que aproximadamente $20 \%$ da variação dos percentuais das escolhas de ímãs com figuras saudáveis em relação aos não saudáveis podem ser creditados às sessões com diferentes vídeos de publicidade entre os grupos. Não houve efeitos significativos $(\mathrm{p}<0,05)$ para as relações da variável independente de medidas repetidas - sessões - com as demais variáveis (covariantes).

Na Tabela 1 observam-se os contrastes entre cada nível da sessão e as variáveis independentes. Na interação entre os níveis 1 e 2 das sessões com os grupos de crianças [F $(1,14)=50,8 ; p<0,05]$ observa-se um $\eta^{2}$ parcial $=29,2 \%$. Isso indica que a variação dos percentuais das escolhas de ímãs com figuras saudáveis em relação aos não saudáveis foram bem explicados por essas variáveis independentes entre esses níveis. Ou seja, as diferenças significativas foram encontradas entre a sessão de vídeos neutros e a primeira sessão com publicidade (saudável ou não saudável), sendo a queda em maior magnitude para o grupo que foi apresentado o vídeo com publicidade não saudável. Já na interação entre os níveis 3 e 4 das sessões com os grupos de crianças [F (1, 14) $=190,1 ; p<0,01]$, observa-se um $\eta^{2}$ parcial $=57,7 \%$. Isso indica que a variação dos percentuais das escolhas de ímãs com figuras saudáveis em relação aos ímãs com figuras não saudáveis foi igualmente bem explicada pelas variáveis independentes. Ou seja, as diferenças significativas foram encontradas entre a segunda sessão com vídeo de publicidade saudável (ou não saudável) e a primeira sessão com vídeo de publicidade não saudável (ou saudável). Essas diferenças tiveram relações opostas, justificando a interação, conforme esperado pelo delineamento experimental. As demais relações não foram significativas $(\mathrm{p}<0,05)$, nem mesmo para as outras covariantes.

Os dados mostram, portanto, efeito dos vídeos, VS e VNS, conduzindo as escolhas de alimentos saudáveis e não saudáveis, respectivamente. Esses resultados confirmam as observações das sessões em que não foram observados efeitos específicos sobre as escolhas dos alimentos quando as crianças tinham sessões antes ou após o lanche escolar.

A Tabela 2 mostra as médias e erro padrão (EP) do percentual de escolha saudável em relação ao de escolha não saudável, por sessão, para cada grupo de crianças. No Grupo VN-VS-VS-VNS-VNS, houve maior média do percentual na sessão $3(\dot{\mathrm{x}}=0,65 ; \mathrm{EP}=0,06)$ e menor média do percentual na sessão $5(\dot{\mathrm{x}}=0,54 ; \mathrm{EP}=0,05)$. No Grupo VN-VNS-VNS-VS-VS, as sessões 1,4 e 5 apresentaram as maiores médias do percentual ( $\dot{\mathrm{x}}=0,63 ; \mathrm{EP}=0,09, \dot{\mathrm{x}}=0,62 ; \mathrm{EP}=0,08, \dot{\mathrm{x}}$ $=0,62 ; \mathrm{EP}=0,06)$ e as sessões 2 e 3 , as menores médias do percentual $(\dot{\mathrm{x}}=0,5 ; \mathrm{EP}=0,09, \dot{\mathrm{x}}=0,51 ; \mathrm{EP}=0,08)$. Portanto, os vídeos alteraram a escolha em até $11 \%$ e $13 \%$, respectivamente, para os grupos VN-VS-VS-VNS-VNS e VN-VNS-VNS-VS-VS.

Na Figura 1 é mostrada a variação da escolha saudável no transcorrer das cinco sessões, para os dois grupos. No Grupo VN-VS-VS-VNS-VNS, apenas com a apresentação da segunda sessão com vídeos saudáveis houve aumento na média do percentual de escolhas saudáveis; na apresentação da segunda sessão com vídeo não saudáveis ocorreu diminuição dessa média para um nível abaixo daquele observado na exposição do vídeo com publicidade neutra. Contudo, conforme os resultados da Tabela 1, as diferenças significa- 
Tabela 1. Tabela de contrastes das variáveis independentes de acordo com a ordem das sessões

\begin{tabular}{|c|c|c|c|c|c|}
\hline Variáveis Independentes & Sessão & $\begin{array}{c}\text { Média } \\
\text { Quadrática }\end{array}$ & $\mathrm{F}$ & Sig. & $\eta^{2}$ parcial \\
\hline \multirow[t]{4}{*}{ Sessão } & Nível 1 vs. Nível 2 & 0,002 & 0,261 & 0,62 & 0,018 \\
\hline & Nível 2 vs. Nível 3 & 0,000 & 0,005 & 0,94 & 0,000 \\
\hline & Nível 3 vs. Nível 4 & 0,006 & 0,888 & 0,36 & 0,060 \\
\hline & Nível 4 vs. Nível 5 & 0,000 & 0,013 & 0,91 & 0,001 \\
\hline \multirow[t]{4}{*}{ Sessão*IMC } & Nível 1 vs. Nível 2 & 0,008 & 0,888 & 0,36 & 0,060 \\
\hline & Nível 2 vs. Nível 3 & 0,005 & 0,176 & 0,68 & 0,012 \\
\hline & Nível 3 vs. Nível 4 & $8,10 \mathrm{E}-04$ & 0,014 & 0,91 & 0,001 \\
\hline & Nível 4 vs. Nível 5 & 0,004 & 0,339 & 0,57 & 0,024 \\
\hline Sessão*Média tempo TV & Nível 1 vs. Nível 2 & 0,002 & 0,196 & 0,66 & 0,014 \\
\hline \multirow[t]{3}{*}{ por semana } & Nível 2 vs. Nível 3 & 0,003 & 0,101 & 0,76 & 0,007 \\
\hline & Nível 3 vs. Nível 4 & 0,008 & $10,218,000$ & 0,29 & 0,080 \\
\hline & Nível 4 vs. Nível 5 & 0,000 & 0,039 & 0,85 & 0,003 \\
\hline \multirow[t]{4}{*}{ Sessão*Idade em meses } & Nível 1 vs. Nível 2 & 0,017 & $10,844,000$ & 0,20 & 0,116 \\
\hline & Nível 2 vs. Nível 3 & 0,023 & 0,854 & 0,37 & 0,057 \\
\hline & Nível 3 vs. Nível 4 & 0,026 & $40,169,000$ & 0,06 & 0,229 \\
\hline & Nível 4 vs. Nível 5 & 0,002 & 0,155 & 0,70 & 0,011 \\
\hline \multirow[t]{4}{*}{ Sessão*Estado nutricional } & Nível 1 vs. Nível 2 & $1,10 \mathrm{E}-05$ & 0,000 & 0,99 & 0,000 \\
\hline & Nível 2 vs. Nível 3 & 0,000 & 0,016 & 0,90 & 0,001 \\
\hline & Nível 3 vs. Nível 4 & $2,03 \mathrm{E}-04$ & 0,004 & 0,95 & 0,000 \\
\hline & Nível 4 vs. Nível 5 & 0,014 & 10,27 & 0,28 & 0,083 \\
\hline \multirow{4}{*}{$\begin{array}{l}\text { Sessão*Grupo de crianças expostas às } \\
\text { publicidades }\end{array}$} & Nível 1 vs. Nível 2 & 0,053 & $50,779,000$ & 0,03 & 0,292 \\
\hline & Nível 2 vs. Nível 3 & 0,019 & 0,704 & 0,42 & 0,048 \\
\hline & Nível 3 vs. Nível 4 & 0,121 & $190,09,000$ & 0,00 & 0,577 \\
\hline & Nível 4 vs. Nível 5 & 0,006 & 0,593 & 0,45 & 0,041 \\
\hline \multirow[t]{4}{*}{ Erro (sessão) } & Nível 1 vs. Nível 2 & 0,009 & & & \\
\hline & Nível 2 vs. Nível 3 & 0,027 & & & \\
\hline & Nível 3 vs. Nível 4 & 0,006 & & & \\
\hline & Nível 4 vs. Nível 5 & 0,011 & & & \\
\hline
\end{tabular}

tivas encontram-se entre as sessões 1 x 2 e 3 x 4 . No Grupo VN-VNS-VNS-VS-VS, apenas a apresentação da primeira sessão com vídeos não saudáveis já gerou diminuição na média do percentual, mantida na segunda apresentação. Da mesma forma, a exposição dos vídeos saudáveis, na primeira sessão, foi seguida por aumento na média quando comparada à exposição do vídeo com publicidade neutra. Novamente, a segunda exposição manteve esses níveis observados com os VS.

\section{Discussão}

Os resultados mostraram um pequeno efeito da variável manipulada (i.e., apresentação de vídeos neutros e com alimentos saudáveis e não saudáveis), ou seja, até $13 \%$, de variação das escolhas de alimentos após a exibição das publicidades. As diferenças significativas foram encontradas na mudança dos vídeos. Do VN para o VS ocorreu, na segunda exposição ao vídeo, maior porcentagem de escolha de alimentos saudáveis. Entretanto, na mudança de VN para VNS foi observada a imediata diminuição nas escolhas saudáveis. 
Tabela 2. Média e erro padrão das escolhas saudáveis entre os grupos

\begin{tabular}{|c|c|c|c|c|c|}
\hline \multirow{2}{*}{ Grupo } & \multirow{2}{*}{ Sessão } & \multirow{2}{*}{$\begin{array}{l}\text { Média das } \\
\text { escolhas }\end{array}$} & \multirow{2}{*}{ Erro Padrão } & \multicolumn{2}{|c|}{$95 \%$ de Intervalo de Confiança } \\
\hline & & & & Limite Inferior & Limite Superior \\
\hline \multirow{5}{*}{ VN-VS-VS-VNS-VNS } & 1 & 0,58 & 0,07 & 0,43 & 0,72 \\
\hline & 2 & 0,57 & 0,07 & 0,42 & 0,73 \\
\hline & 3 & 0,65 & 0,06 & 0,52 & 0,79 \\
\hline & 4 & 0,58 & 0,07 & 0,44 & 0,72 \\
\hline & 5 & 0,54 & 0,05 & 0,44 & 0,64 \\
\hline \multirow{5}{*}{ VN-VNS-VNS-VS-VS } & 1 & 0,63 & 0,09 & 0,44 & 0,81 \\
\hline & 2 & 0,50 & 0,09 & 0,30 & 0,69 \\
\hline & 3 & 0,51 & 0,08 & 0,33 & 0,68 \\
\hline & 4 & 0,62 & 0,08 & 0,44 & 0,80 \\
\hline & 5 & 0,62 & 0,06 & 0,49 & 0,75 \\
\hline
\end{tabular}

Esse efeito também foi observado na mudança do VS para o VNS, em que houve a diminuição das escolhas saudáveis, e do VNS para VS, em que ocorreu o aumento dos itens saudáveis. Os vídeos com publicidade de alimentos saudáveis (VS) e não saudáveis (VNS) podem ter exercido controle discriminativo sobre a escolha dos alimentos (Foxall, 2004), pois em geral, foram observadas escolhas dos alimentos da publicidade após a exibição da mesma, tanto para alimentos saudáveis, como não saudáveis.

Assim, os resultados com crianças de 7 a 9 anos são consistentes com os de Peterson et al. (1984) e Dixon et al. (2007) com crianças na idade de 5 a 6 anos e 10 a 11 anos, respectivamente. Esses dois estudos destacam: 1) alimentos saudáveis foram selecionados após um programa voltado

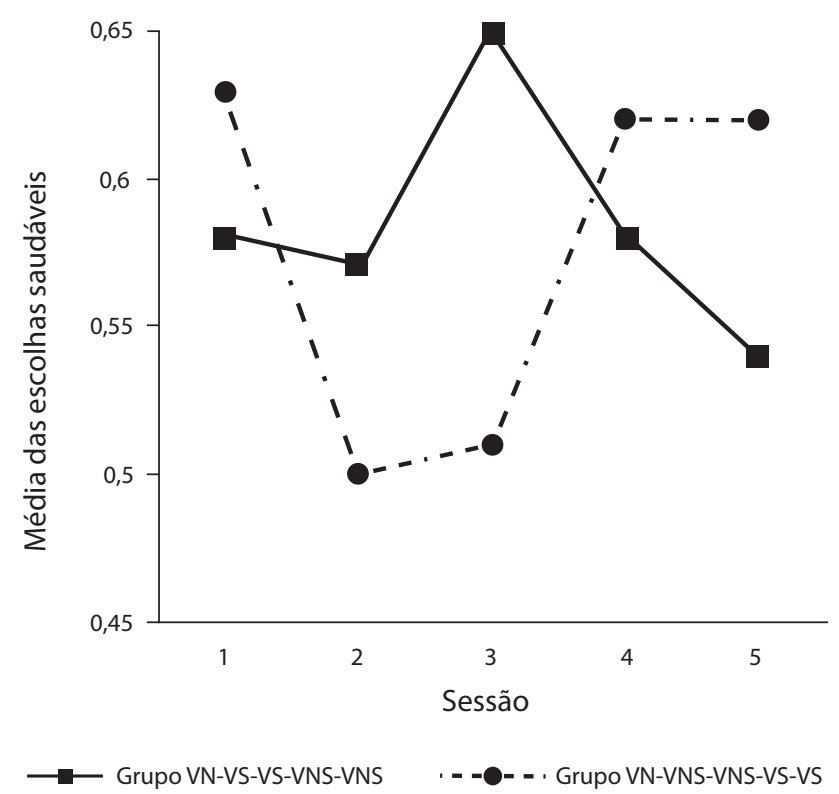

Figura 1. Média das escolhas saudáveis intra e entre sujeitos nas sessões. para nutrição e saúde (Peterson et al., 1984); 2) efeitos da exposição a vídeos não saudáveis sobre as escolhas de itens não saudáveis, assim como ocorrência de comportamentos voltados para alimentos não saudáveis decorrentes das publicidades desses alimentos (Dixon et al., 2007).

Todos os alimentos que não foram apresentados nas publicidades da pesquisa, foram também escolhidos e podem refletir o efeito da história alimentar e da publicidade de alimentos no ambiente natural. Portanto, a exposição à publicidade da pesquisa pode somar-se à exposição à publicidade de alimentos não saudáveis no ambiente natural. Da mesma forma que a criança escolheu alimentos que não estavam presentes nas publicidades, ela poderia escolher produtos cujas marcas fossem conhecidas por ela. Por isso, a seleção de publicidades desconhecidas pela criança, como foi feito no presente estudo, representa um controle experimental necessário para que as escolhas dos alimentos sejam relacionadas à publicidade programada no ambiente experimental, além de não promover uma marca que possa ser consumida em seu ambiente natural.

Os dados obtidos quanto ao consumo de frutas e verduras e do tempo de exposição à televisão mostraram semelhança com aqueles do estudo de Boynton-Jarret et al. (2003). Cerca de $50 \%$ das crianças não consomem estes alimentos frequentemente e apresentaram uma alta exposição à mídia televisiva. A substituição desses itens por alimentos não saudáveis da publicidade, pode estar contribuindo para o aumento do peso, corroborando os resultados encontrados em Zimmerman e Bell (2010).

Assim, o controle da exposição à televisão é sugerido em intervenções voltadas para a redução da obesidade (Robinson, 1999; Veerman, Van Beeck, Barendregt, \& Mackenbach, 2009). Considerando que, em geral, os alimentos divulgados na publicidade apresentam baixa qualidade nutricional, a Academia Americana de Pediatria (AAP) recomenda às famílias que limitem o tempo diante da televisão, internet $\mathrm{e}$ filmes a, no máximo, duas horas diárias, além de incentivar a 
educação nutricional (Batada, Seitz, Wootan, \& Story, 2008). As crianças da presente pesquisa apresentaram uma média de quatro diárias de exposição à televisão, sendo, portanto, necessária a redução da mesma ou o aumento da exposição à publicidade de alimentos saudáveis na mídia.

A redução da exposição à mídia televisiva deve ser acompanhada da construção de novos repertórios comportamentais voltados para brincadeiras no dia a dia que substituirão o tempo de exposição, e, ainda, novas práticas de alimentação que envolvem desde o que é comprado, até a organização dos alimentos em casa de modo a facilitar o acesso aos alimentos saudáveis, a oferta dos mesmos nas mesas de refeição e o consumo de alimento por parte dos principais cuidadores, que são o modelo para a criança (Hurtsi, 1999). Buijzen (2009) e Buijzen, Schuurman e Bomhof (2008) destacam a necessidade da mediação dos pais diante de conteúdos e imagens veiculadas pela mídia, desenvolvendo os valores da cultura familiar, quando a mídia sinaliza outros valores.

Apesar de grande parte das crianças da pesquisa terem sido acompanhadas durante exposição à televisão, é importante o desenvolvimento de uma abordagem crítica do conteúdo e imagens veiculados na mídia (Porto, 2011) de modo a selecionar os efeitos da publicidade, enfraquecendo valores voltados para os alimentos não saudáveis e fortalecendo os alimentos saudáveis ou práticas saudáveis de alimentação (Hindin, Contento, \& Gussow, 2004).

A promoção de alimentos saudáveis pelas famílias, escolas, cantinas, mídias, envolvendo profissionais de saúde e entidades governamentais mostra-se urgente. Esses valores do profissional de saúde, da família, dos educadores são úteis e potencialmente efetivos na orientação de crianças às práticas alimentares saudáveis (Vasconcelos, 2006). Entretanto, isso pode não estar ocorrendo com uma frequência adequada, considerando o aumento no consumo de alimentos não saudáveis e no aumento do sobrepeso e obesidade infantil, enquanto os valores da mídia podem estar ocorrendo simultaneamente e em alta frequência (Veerman et al., 2009; Zimmerman \& Bell, 2010).

A regulamentação da publicidade poderia contribuir para diminuir os estímulos que favorecem o consumo de alimentos não saudáveis pelas crianças, tanto na mídia televisiva, quanto na internet e revistas infantis. Por outro lado, facilitar e favorecer a publicidade de alimentos saudáveis em todas as mídias, assim como em programas de educação nutricional podem contribuir para a adoção de práticas alimentares mais saudáveis (Byrd-Bredbenner \& Grasso, 2000; Dixon et al., 2007; Peterson et al., 1984).

Contudo, um impasse tem dificultado a regulamentação da publicidade no Brasil. De um lado está a proteção das crianças contra a publicidade com efeitos adversos direcionada ao público infantil e de outro, a resistência das empresas para a regulamentação da publicidade pautando-se na liberdade de expressão do setor econômico. Estudos voltados para a publicidade e escolha de alimentos podem contribuir para a limitação da publicidade de alimentos não saudáveis para as crianças ou para a promoção da alimentação saudável na mídia. Replicações futuras podem programar um número maior de sessões, com a utilização de um índice de estabilidade das escolhas; apresentar maior tempo da publicidade por sessão; limitar a quantidade de alimentos a serem escolhidos, em relação ao que foi disponibilizado neste estudo (36 alternativas). Alimentos reais, previamente autorizados pelos responsáveis, poderiam também ser introduzidos em novos estudos. Ademais, o controle do horário do experimento e do horário das refeições reais da criança constituem-se, também, em variáveis a serem investigadas sistematicamente. O conhecimento gerado por esses estudos podem fornecer mais dados para realizar planejamentos culturais. Equipes interdisciplinares - nutricionistas, analistas do comportamento, médicos, professores e repórteres podem programar intervenções em ampla escala de forma a alertar, prevenir e intervir em rotinas de risco para as crianças com alta exposição às mídias de tela e com alto consumo de alimentos calóricos, industrializados e de baixo valor nutritivo (e.g., Bucht \& Feilitzen, 2002; Vasconcelos, 2006, 2010).

\section{Referências}

Agência Nacional de Vigilância Sanitária - ANVISA (2007). Alimentação saudável: fique esperto. Retrieved from http:// www.anvisa.gov.br/propaganda/alimento_saudavel_gprop_ web.pdf

Almeida, S. S., Nascimento, P .C .B .D., \& Quaioti, T. C. B. (2002). Quantidade e qualidade de produtos alimentícios anunciados na televisão brasileira. Revista de Saúde Pública, 36, 353-355.

Andersen, R. E., Crespo, C. J., Bartlett, S. J., Cheskin, L. J., \& Pratt, M. (1998). Relatioship of physical activity and television watching with body weight and level of fatness among children: Results from the Third National Health and Nutrition Examination Survey. The Journal of the American Medical Association, 25, 938-942.

Batada, A., Seitz, M. D., Wootan, M. G., \& Story, M. (2008). Nine out of 10 food advertisements shown during saturday morning children's television programming are for foods high in fat, sodium, or added sugars, or low in nutrients. Journal of American Dietetic Association, 108, 673-678.

Borzekowski, D. L. G., \& Robinson, T. N. (2001). The 30-second effect: An experiment revealing the impact of television commercials on food preferences of preschoolers. Journal of the American Dietetic Association, 101, 42-46.

Boynton-Jarrett, R., Thomas, T. N ., Peterson, K. E., Wiecha, J., Sobol, A. M., \& Gortmaker, S. L. (2003). Impact of television viewing patterns on fruit and vegetable consumption among adolescents. Pediatrics, 112, 1321-1326.

Bucht, C., \& Feilitzen, C. V. (2002). Perspectivas sobre a criança e a mídia. Brasília: UNESCO, SEDH/ Ministério da Justiça.

Buijzen, M. (2009). The effectiveness of parental communication in modifying the relation between food advertising and children's consumption behavior. British Journal of Developmental Psychology, 27, 105-121.

Buijzen, M., Schuurman, J., \& Bomhof, E. (2008) Associations between children's television advertising exposure and their food consumption patterns: A household diary-survey study. Appetite, 50, 231-239.

Byrd-Bredbenner, C., \& Grasso, D. (2000). What is televisión trying to make children swallow? Content analysis of the nutrition information in prime-time advertisements. Journal of Nutrition Education, 32, 187-195. 
Coelho, F. U. (1994). O empresário e os direitos do consumidor. São Paulo: Saraiva.

Cohen, D., Benjamin, A., \& Vasconcellos, A. H. (2005). Código brasileiro de defesa do consumidor comentado pelos autores do anteprojeto. Rio de Janeiro: Forense Universitária.

Conde, W. L., \& Monteiro, C. A. (2006). Valores críticos do índice de massa corporal para classificação do estado nutricional de crianças e adolescentes brasileiros. Jornal de Pediatria, 82, 266-272.

Dixon, H. G., Scully, M. L., Wakefield, M. A., White, V. M., \& Crawford, D. A. (2007). The effects of television advertisements for junk food versus nutritious food on children's food attitudes and preferences. Social Science \& Medicine, 65, 1311-1323.

Foxall, G. R. (2004). Consumer psychology in behavioural perspective. Washington, DC: Beard Books.

Groebel, J. (2002). Acesso à mídia e uso da mídia entre crianças de 12 anos no mundo. In U. Carlsson, \& C. V. Feilitzen (Eds.), A criança e a midia: imagem, educação e participação (pp. 6976) (D. A. Azevedo \& M. E. S. Matar, Trads.). Brasília: Unesco.

Halford, J. C. G., Gillespie, J., Brown, V., Pontin, E. E., \& Dovey, T. M. (2004). Effect of television advertisements for foods on food consumption in children. Appetite, 42, 221-225.

Halford, J. C. G., Boyland, E. J., Hughes, G. M., Stacey, L., McKean, S., \& Dovey, T. M. (2007). Beyond-brand effect of television food advertisements on food choice in children: The effects of weight status. Public Health Nutrition, 11, 897-904.

Halpern, G. (2003). Comerciais veiculados em programação infanto-juvenil de canais abertos de TV e sua relação com a escolh a de alimentos em amostra de escolares. Dissertação de mestrado não publicada, Universidade Federal de São Paulo, São Paulo.

Hindin, T. J., Contento, I. R., \& Gussow, J. D. (2004). A media literacy nutrition education curriculum for Head Start Parents about the effects of television advertising on their children's food requests. Journal of the American Dietetic Association, 104, 192-198.

Hursti, U. K. (1999). Factors influencing children's food choice. The Finnish Medical Society Duodecim, 31(1), 26-32.

IBGE (2008). Pesquisa Nacional por Amostra de Domicílio Síntese dos indicadores 2007. Retrieved from http://www. ibge.gov.br/home/estatistica/populacao/trabalhoerendimento/ pnad2007/sintesepnad2007.pdf

Jago, R., Baranowski, T., Baranowski, J. C., Thompson, D., \& Greaves, K. A. (2005). BMI from 3-6y of age is predicted by TV viewing and physical activity, not diet. International of Obesity, 29, 557-564.

Mendonça, C. P., \& Anjos, L .A. (2004). Aspectos das práticas alimentares e da atividade física como determinantes do crescimento do sobrepeso/obesidade no Brasil. Cadernos de Saúde Pública, 20, 698-709.

Ministério da Justiça (2009). Classificação indicativa. Informação e liberdade de escolha. Brasília: Artecor.

Monteiro, R. A. (2009). Influência de aspectos psicossociais e situacionais sobre a escolha alimentari infantil. Tese de doutorado não publicada, Universidade de Brasília, Brasília.

Nascimento, P. C. B. D. (2006). A influência da televisão nos hábitos alimentares de crianças e adolescentes. Tese de doutorado não publicada, Universidade de São Paulo, Ribeirão Preto.

Nunes, R., (2011). Publicidade e consumo. Revista Consulex, 349, 24-27.
Peterson, P. E., Jeffrey, D. B., Bridgwater, C. A., \& Dawson, B. (1984). How pronutrition television programming affects children's dietary habits. Developmental Psychology, 20, 55-63.

Porto, R. B. (2011). Conceitos comportamentais em filme de publicidade: Aplicação de técnicas que descrevem a mensagem persuasiva. Revista de Psicologia, 42(3), 310-318.

Porto, R. B., \& Oliveira-Castro, J. M. (2013). Say-do correspondence in brand choice: Interaction effects of past and current contingencies. The Psychological Record, 63, 345-362.

Radnitz, C., Byrne, S., Goldman, R., Sparks, M., Gantshar, M., \& Tung, K. (2009). Food cues in children's television programs. Appetite, 52, 230-233.

Robinson, T. N. (1999). Reducing children's television viewing to prevent obesity: A randomized controlled trial. Journal of American Medical Association, 282, 1561-1567.

Robinson, T. N., Borzekowski, D. L. G., Matheson, D. M., \& Kraemer, H. C. (2007). Effects of fast food branding on young children's taste preferences. Archives of Pediatric \& Adolescent Medicine, 161, 792-797.

Vasconcelos, L. A. (2006). A mídia e o desenvolvimento de crianças e jovens. In H. J. Guilhardi, \& N. C. Aguirre (Eds.), Sobre comportamento e cognição (vol. 17, pp. 357-365). Santo André, SP: Esetec.

Vasconcelos, L. A. (2010). Práticas culturais envolvendo a criança e a mídia: Interface entre comunicação e a Análise do Comportamento. In M. M. C., Hübner, M. R. Garcia, P. R. Abreu, E. N. P. Cillo, \& P. B. Faleiros (Eds.), Sobre comportamento e cognição: análise experimental do comportamento, cultura, questões conceituais e filosóficas (vol. 25, pp. 129-136). Santo André, SP: Esetec.

Veerman, J. L., Van Beeck, E. F., Barendregt, J. J., \& Mackenbach, J. P. (2009). By how much would limiting TV food advertising reduce childhood obesity? European Journal of Public Health, 19, 365-369.

Vilaça, E. L., Paixão, H. H., \& Pordeus, I. A. (1997). A mídia e as tendências de consumo de produtos contendo sacarose por crianças e adolescentes. Arquivos em Odontologia, 33, 15-24.

Zimmerman, F. J., \& Bell, J. F. (2010). Associations of television content type and obesity in children. American Journal of Public Health, 100, 334-340.

Recebido em 21.11.2010

Primeira decisão editorial em 05.04.2013

Versão final em 16.09.2013

Aceito em 23.09.2013 